\title{
The endoscopic evaluation of gastritis, gastric remnant residue, and the incidence of secondary cancer after pylorus-preserving and transverse gastrectomies
}

\author{
Hideki Nagano $^{1}$, Shigekazu Ohyama ${ }^{1}$, Yoshiniro Sakamoto ${ }^{1}$, Keitchiro Ohta ${ }^{1}$, Toshiharu Yamaguchi ${ }^{1}$, \\ Tetsuichiro $\mathrm{Muto}^{1}$, and Akio Yamaguchi ${ }^{2}$ \\ ${ }^{1}$ Department of Surgery, Cancer Institute Hospital, 1-37-1 Kami-ikebukuro, Toshima-ku, Tokyo 170-8455, Japan \\ ${ }^{2}$ First Department of Surgery, Faculty of Medicine, Fukui Medical University, Fukui, Japan
}

\begin{abstract}
Background. Pylorus-preserving gastrectomy (PPG) and transverse gastrectomy (TrG) have been accepted as functionpreserving procedures for node-negative early gastric cancer. It is believed that a better quality of life is guaranteed after PPG or TrG compared to that after distal subtotal gastrectomy (DSG) with Billroth type-I reconstruction. However, objective evaluations of the gastric remnant following gastrectomy have not been widely reported, and the real advantages and disadvantages of PPG or TrG over DSG remain unclear. Moreover, the risk of secondary cancer after PPG or TrG is uncertain.

Methods. Between 1991 and 2000, 834 DSGs were carried out in our institute for preoperatively diagnosed patients with early gastric cancer. The degree of residual gastritis and the amount of diet residue in the gastric remnant were evaluated by annual gastrointestinal endoscopic investigations prospectively for 72 patients after PPG, 95 patients after TrG, and 60 patients after DSG. These analyses were performed using the RGB classification (residue, gastritis, bile). The incidence of disease greater than or equal to grade 2 was calculated, and the time trends of the incidence for each procedure were also studied for 3 years after gastrectomy. In addition, secondary cancer cases in the gastric remnant mucosa were checked for each procedure during this period, and the incidence of secondary cancer after each operation was calculated.

Results. The incidence of gastritis, of grade 2 or more, found in the gastric remnant was significantly lower after PPG (1.4\%) and TrG (2.1\%) than after DSG (43.3\%). However, the incidence of moderate or greater residue in the gastric remnant, grade 2 or more, was significantly higher after PPG $(45.8 \%)$ and TrG $(40.0 \%)$ than after DSG $(11.7 \%)$. The analysis of time trends of gastritis and diet residue reflected the significant advantage or disadvantage for each procedure 1 year after surgery. The analysis also included these factors without consideration of elapsed time following surgery. Two patients after PPG $(\mathbf{2 . 8} \%)$ and three patients after TrG
\end{abstract}

Offprint requests to: S. Ohyama

Received: September 8, 2003 / Accepted: January 6, 2004
(3.2\%) developed secondary cancer in the gastric remnant. No DSG-treated patient showed new cancer genesis in the remaining stomach.

Conclusion. PPG and TrG have the advantage over DSG in preventing postoperative gastritis in the gastric remnant. On the other hand, moderate or greater diet residue in the gastric remnant is more common after PPG or TrG than after DSG. For the risk of carcinogenesis in the remnant gastric mucosa, we could not conclude that there was any apparent difference between these range-limited gastrectomies and conventional DSG. Further study is necessary to determine the significant advantages and disadvantages of using PPG or TrG.

Key words Pylorus-preserving gastrectomy · Transverse gastrectomy - Distal subtotal gastrectomy - Residual gastritis · Diet residue $\cdot$ Secondary cancer

\section{Introduction}

Pylorus-preserving gastrectomy (PPG) and transverse gastrectomy ( $\mathrm{TrG})$ have been accepted as functionpreserving procedures, especially in Japan [1-13]. Excellent postoperative quality of life (QOL), longterm survival, and low rates of recurrence have been associated with these procedures. However, the obvious advantages of limited gastrectomy have not been systematically evaluated. After these operations, we have often encountered patients complaining of static symptoms, probably emanating from the prolonged emptying time of the gastric remnant. The significant advantages of these limited gastrectomies compared to conventional distal subtotal gastrectomy (DSG) with Billroth type-I reconstruction remain unclear.

Moreover, we wanted to determine the long-term postoperative risk of secondary cancer in the remaining stomach. The mucosal environment would appear to be dissimilar in these limited gastrectomies and in DSG, but we do not yet have enough information about the mucosal status of these range-limited gastrectomies. 
In this study, we attempted to elucidate the advantages and disadvantages of PPG and TrG over conventional DSG by the annual measurement of the degree of residual gastritis and the amount of diet residue in the gastric remnant for up to 3 years following the operation, using the RGB classification (residue, gastritis, bile) [14]. We also attempted to evaluate secondary cancer cases after these procedures to elucidate the risk of carcinogenesis in the residual gastric mucosa for each procedure.

\section{Patients and methods}

Between January 1991 and December 2000, surgical operations were performed in 2845 patients with primary gastric cancer. There were 1505 patients with early gastric cancers. PPG, TrG, and DSG were performed in 112, 120, and 834 patients, respectively. A total of 72 PPG patients and $95 \mathrm{TrG}$ patients underwent postoperative gastrointestinal endoscopic investigation. During this period, 60 patients who underwent DSG for early gastric cancer were randomly selected for comparison (Table 1). In the DSG group, the celiac branch of the vagal nerve was preserved in all patients. We used endoscopy to assess the degree of residual gastritis and the amount of diet residue in these patients, using the RGB classification.

In addition, the gastric remnant mucosa in patients with secondary cancer was assessed for each procedure during this period, and the incidence of secondary cancer after each operation was calculated.

\section{Indications and surgical procedures}

PPG was carried out in patients with early gastric cancer that remained in the mucosal or submucosal layer, or in the middle or lower part of the stomach, or at least $5 \mathrm{~cm}$ from the pylorus. The hepatic and pyloric branches of the vagal nerve and right gastric vessels were preserved, but lymph node dissection along the right gastric vessels was carried out. The infrapyloric artery, if present, was preserved [1,2]. The left gastric artery was ligated, and this preserved the celiac branch of the vagal nerve. The lesser curvature of the oral gastric remnant was halfclosed, leaving the anastomotic site size-matched for the distal gastric remnant. Gastro-gastro anastomoses were completed in a layer-to-layer fashion.

TrG was performed in patients with early gastric cancer confined to the mucosal layer in the middle part of the stomach. These patients also had lesions containing a microscopically differentiated structure (tub1 or tub2) that spread superficially within $2 \mathrm{~cm}$ in size and that contained a preoperative node-negative status. The hepatic branch and the celiac branch of the vagal nerve were preserved, while the left gastric artery was ligated at its descending region along the lesser curvature. Lymph node dissection was limited near the tumor. Gastro-gastro anastomoses were also carried out in a layer-to-layer fashion.

\section{Evaluation of the gastric remnant}

Every patient received gastrointestinal endoscopy at least once within 2 years after the gastrectomy. The findings of the residual stomach were prospectively evaluated every year for 3 years after the operation and analyzed for the degree of residual gastritis and the amount of diet residue in the gastric remnant, using the RGB classification (Table 2). The incidence of gastritis or residue of grade 2 or greater was calculated. Variations in the incidence rate of grade 2 or greater gastritis or residue were also analyzed for 3 years.

\section{Statistical analyses}

For statistical analyses, the Mann-Whitney $U$-test was used, and a $P$ value less than 0.05 was considered significant.

Table 1. Characteristics of patients in the three groups

\begin{tabular}{|c|c|c|c|c|}
\hline & $\operatorname{TrG}$ & PPG & DSG & $P$ value \\
\hline Male/Female & $57 / 38$ & $35 / 37$ & $39 / 21$ & NS \\
\hline Age (years) & $60.0 \pm 10.2(1 *)$ & $55.9 \pm 10.9(1 *)$ & $57.3 \pm 11.7$ & $1 * P=0.012$ \\
\hline \multicolumn{5}{|c|}{ Range of LN dissection } \\
\hline D0 & 921 & 421 & 111 & $2 * P<0.001$ \\
\hline D1 & $(2 * ; 3 *)$ & $(2 * ; 4 *)$ & \begin{tabular}{l|l}
5 & $\left(3 * ; 4^{*}\right)$
\end{tabular} & $3 * P<0.001$ \\
\hline D2 & 0 & 23 & $44]$ & ${ }^{4 *} P<0.001$ \\
\hline \multicolumn{5}{|c|}{ Stage of disease } \\
\hline IA & $95]$ & 70 & $56]\left(5^{*}\right)$ & $5 * P=0.011$ \\
\hline IB & $0]$ & 2 & $4]$ & \\
\hline
\end{tabular}

TrG, transverse gastrectomy; PPG, pylorus-preserving gastrectomy; DSG, distal subtotal gastrectomy; LN, lymph node; NS, not significant 


\section{Results}

\section{Degree of residual gastritis}

Only one patient presented with residual gastritis greater than or equal to grade 2 in the RGB classification after PPG $(1.4 \%)$ and $\operatorname{TrG}(2.1 \%)$, whereas DSG patients had a much higher incidence $(43.3 \%$; Fig. 1). DSG patients had the greatest improvement in the incidence of gastritis greater than or equal to grade 2 as time progressed. However, even with these improved measures, the DSG group had significantly higher rates compared to the PPG or TrG groups (Fig. 2A).

Table 2. Residue, gastritis, bile (RGB) classification

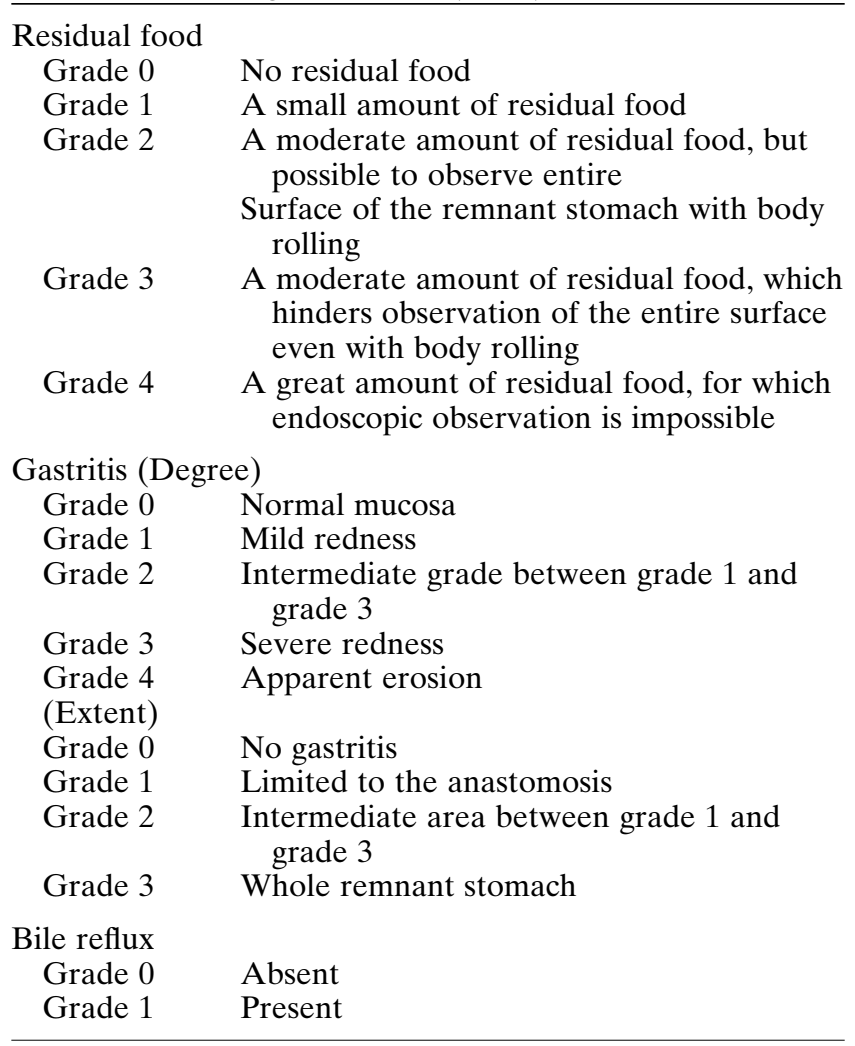

\section{Amount of diet residue in the gastric remnant}

The DSG group had significant improvement in the amount of diet residue in the gastric remnant compared with the PPG or TrG groups $(11.7 \%, 45.8 \%$, and $40.0 \%$, respectively; Fig. 1). On time trend analysis, the DSG group had a lower incidence of diet residue equal to or greater than grade 2 compared with the PPG or TrG groups for each year (Fig. 2B). No significant improvements in the amount of diet residue were found in any of the groups, and the PPG and TrG groups had persistently significant amounts of diet residue.

\section{Secondary cancer in the gastric remnant}

Two patients developed secondary gastric cancer in the gastric remnant following PPG, and three patients developed secondary gastric cancer after $\operatorname{TrG}$ (Table 3). In the DSG group, secondary cancer cases were not detected during this period.

\section{Discussion}

DSG is a standard operative procedure for gastric cancer [15]. During the follow-up of DSG-treated patients, we encountered patients suffering several symptoms, such as dumping syndrome, reflux esophagitis, and impaired weight gain. Under such circumstances, various function-preserving surgical procedures have been attempted for patients with early gastric cancer $[3,4,16]$. Maki et al. [5] developed PPG as a function-preserving procedure for gastric ulcer in 1967.

Since Kodama and Koyama [4] first reported the indication of PPG for early gastric cancer in 1991, we have used PPG in the preservation of the pyloric branch of the vagal nerve as one option for node-negative early gastric cancer surgery.

Maki et al. [5] recommended preserving approximately $1.5 \mathrm{~cm}$ proximal to the pylorus. This was based on information gained from an experimental study using dogs. Several institutes have reported PPG cases
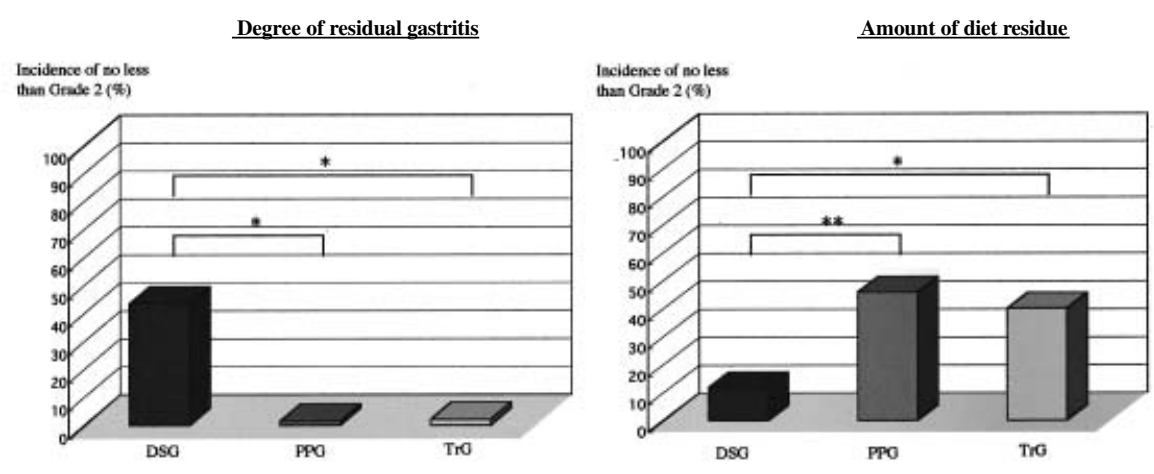

Fig. 1. Evaluation of the degree of residual gastritis and the amount of diet residue among three groups of patients, who underwent pylorus-preserving gastrectomy $(P P G)$, transverse gastrectomy $(\operatorname{Tr} G)$, or distal subtotal gastrectomy $(D S G)$. The incidence of gastritis of no less than grade 2 was significantly lower after PPG and TrG than after DSG, and the incidence of residue no less than grade 2 was significantly higher after PPG and TrG than after DSG $(* P<0.0001 ; * * P=0.0020)$ 


\section{A. Residual gastritis}

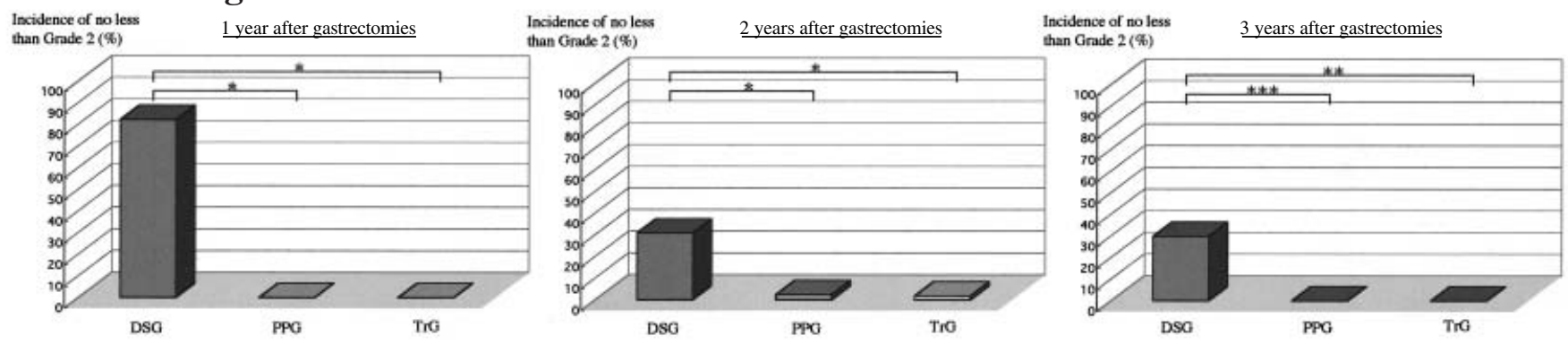

\section{B. Amount of diet residue}
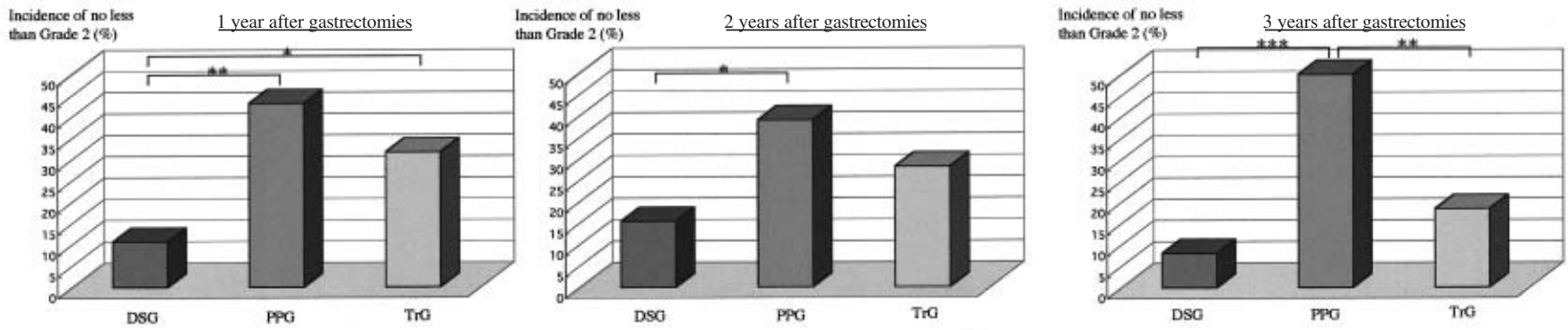

Fig. 2. A The incidence of residual gastritis of no less than grade 2 was significantly higher in DSG cases compared with PPG or TrG. This trend had been observed since the first year after operation. This trend persisted for 3 years. DSG patients demonstrated improvements in gastritis as time progressed $(* P<0.001 ; * * P<0.002 ; * * * P<0.02)$. B For each year, the

DSG group presented a lower incidence of diet residue of no less than grade 2 compared with the PPG or the TrG group. For each group, significant improvements in incidence rates were not observed, and the PPG and TrG (especially PPG) groups demonstrated persistent amounts of diet residue $(* P<0.05 ; * * P<0.01 ; * * * P<0.001)$

Table 3. Five cases of residual gastric cancer after PPG and transverse gastrectomy (TrG)

\begin{tabular}{|c|c|c|c|c|c|c|c|c|c|}
\hline $\begin{array}{l}\text { Case } \\
\text { no. }\end{array}$ & $\begin{array}{c}\text { Age (in years, } \\
\text { at first } \\
\text { operation }\end{array}$ & Sex & $\begin{array}{c}\text { Prior } \\
\text { operation }\end{array}$ & $\begin{array}{c}\text { Residual } \\
\text { gastritis } \\
\text { (grade) }\end{array}$ & $\begin{array}{l}\text { Amount } \\
\text { of residue } \\
\text { (grade) }\end{array}$ & $\begin{array}{l}\text { Site of } \\
\text { second } \\
\text { cancer }\end{array}$ & $\begin{array}{l}\text { Period to } \\
\text { second } \\
\text { cancer }\end{array}$ & $\begin{array}{c}\text { Macroscopic } \\
\text { type of second } \\
\text { cancer }\end{array}$ & $\begin{array}{l}\text { Pathological } \\
\text { type of second } \\
\text { cancer }\end{array}$ \\
\hline 1 & 82 & $\mathrm{~F}$ & PPG & 1 & 3 & Anal side & 7 Years & IIc & tub1 \\
\hline 2 & 53 & $\mathrm{~F}$ & PPG & 0 & 2 & Oral side & 6 Years & I & tub1 \\
\hline 3 & 64 & M & TrG & 0 & 0 & Anal side & 5 Years & $\mathrm{IIa}+\mathrm{IIc}$ & tub1 \\
\hline 4 & 61 & M & $\operatorname{TrG}$ & 4 & 2 & Anal side & 8 Years & 4 & por2 \\
\hline 5 & 56 & $\mathrm{M}$ & $\operatorname{TrG}$ & 1 & 0 & Oral side & $\begin{array}{l}1 \text { Year } \\
\text { and } \\
4 \text { months }\end{array}$ & IIc & sig \\
\hline
\end{tabular}

where the remaining $1.5 \mathrm{~cm}$ antral portion is transected in Maki's fashion [1,2,6-10]. However, Nakane et al. [17] demonstrated the advantage of PPG involving a transection $2.5 \mathrm{~cm}$ proximal to the pylorus. The advantage of this approach, compared with a $1.5-\mathrm{cm}$ transection, is evident in improved postprandial symptoms, food intake, body-weight recovery, and gastric emptying. In our study, all PPG patients had transections $3.0 \mathrm{~cm}$ proximal to the pylorus, so that $2.5 \mathrm{~cm}$ remained after anastomosis.

Our group first applied TrG with nerve preservation for patients with early gastric cancer. This preserves the antral part of the stomach to at least $3 \mathrm{~cm}$ in length. When applied to a more limited lesion, the patient can expect the same or a better QOL than with PPG.

Although these procedures ( $\mathrm{TrG}$ and PPG) are less invasive, the cure rate of gastric cancers must not be compromised. Regional lymph nodes were excised in addition to conventional resection. In addition, the antropyloric region, nerves, and postoperative digestive functions were preserved $[2,10]$. We could not find a difference in the long-term survival between the gastriccancer groups receiving function-preserving procedures and those receiving conventional DSG matched at each 
stage (data not shown). These less invasive procedures did not significantly affect the cure rate.

In patients with segmental gastric resection, the pylorus and the pyloric branch of the vagal nerve were preserved to coordinate the function of the pylorus. Dumping syndrome and reflux gastritis rates [18-20] were consistent with this type of surgery. Many authors have reported improved QOL after PPG compared with DSG [1,6,11].

Although these procedures dramatically reduced symptoms derived from dumping or gastritis, patients often complained of static symptoms caused by the prolonged emptying time of the gastric remnant $[8,12,13]$. Recently, scintigraphic comparisons of the gastric emptying rates of solids and liquids between patients receiving PPG and those receiving classical DSG have been reported [9]. A dual-isotope assay revealed that the liquid gastric emptying was accelerated in both PPG and DSG during the early postprandial period, whereas solid emptying was accelerated only in DSG.

Some authors have reported that, in patients who have undergone PPG, postoperative endoscopic study revealed a moderate or great amount of residual food in the gastric remnant $[1,6,7,11]$, but the status of the gastric remnant has not been well documented. For endoscopic evaluations of the remnant stomach following gastrectomy, Kubo et al. [14] proposed an easy and quantitative classification (RGB; residue, gastritis, bile). This classification seems to be a useful way to describe the residual gastric findings. In this study, we applied this RGB classification to a quantitative analysis of the degree of residual gastritis and the amount of diet residue after PPG and TrG. In this study, we investigated the annual endoscopic findings and attempted to elucidate not only their temporal condition, but also their time trends. If the scores show steady improvement over time, then the quality of the operation should not be evaluated with the temporal worst scores given at the first time. Therefore, the variation of the score and the time trend should be analyzed. In this study, not all patients underwent annual examinations for 3 years, but the approximate trends could be evaluated. As expected, the degree of residual gastritis was significantly lower after PPG or TrG than after DSG, probably owing to the reduced reflux of duodenal juice. The reflux of bile or pancreatic juice into the gastric remnant may increase the risk of carcinogenesis through remnant mucosal cell damage or acceleration of cell proliferation in the gastric mucosa [8,21-23]. From this aspect, DSG may engender a higher prevalence rate for secondary gastric cancer after a long period than either PPG or TrG.

On the other hand, longer stasis of the gastric residue may generate carcinogenic substances such as $\mathrm{N}$-nitroso-complexes, especially in bile juice [8]. Thus, the residual gastric mucosa would be exposed to these harmful substances for a longer period of time.

It has been reported that the rate of synchronous multiple early gastric cancer ranged from $9.0 \%$ to $11.7 \%$ [24-26] The most prevalent location of these accessory lesions is adjacent to the primary lesion in the middle or lower third of the stomach. Moreover, intestinal metaplasia has been regarded as the major cause of multicentric carcinogenesis of the stomach [27-31]. However, the reported preoperative diagnosis of accessory lesions was insufficient in these studies [24,25].

Range-limited gastrectomies, such as PPG or TrG, leave the antral part with a high risk of carcinogenesis and leave accessory lesions overlooked. For these procedures, careful preoperative diagnosis and postoperative follow-up seem to be important to detect simultaneous accessory lesions or newly developed lesions.

In our study, two patients with PPG and three with TrG developed secondary gastric cancer (Table 3). In the DSG group, we did not encounter secondary cancer cases, but Takeda et al. [32] concluded that the incidence of early gastric cancer in the remnant stomach after conventional DSG was $1.8 \%$ (15/845). Their study was not based on follow-up study after gastrectomies, so we could not directly compare their data with our findings. However, considering their data, it seems that the actual incidence of carcinogenesis in the DSG-treated residual mucosa in our patients would not deviate far from their data. We could not conclude that the PPG- or TrG-treated patients had a higher risk of secondary cancer compared with the DSG group. The association of carcinogenesis in the remnant gastric mucosa with range-limited gastrectomies is uncertain.

PPG and TrG procedures are associated with a lower incidence of dumping syndrome and gastritis and worse emptying time than DSG. Further study will be necessary to reveal the real advantages and disadvantages of PPG and TrG compared to conventional DSG. This issue should be considered from various viewpoints, such gastric cancer cure rate, postoperative symptoms, body weight gain, nutritional status, and the risk of carcinogenesis in the remnant gastric mucosa.

\section{References}

1. Kodama M, Koyama K, Chida T, Arakawa A, Tur G. Early postoperative evaluation of pylorus-preserving gastrectomy for gastric cancer. World J Surg 1995;19:456-61.

2. Sawai K, Takahashi T, Fujioka $T$, Minato $H$, Taniguchi $H$, Yamaguchi T. Pylorus-preserving gastrectomy with radial lymph node dissection based on anatomical variations of the infrapyloric artery. Am J Surg 1995;170:285-8.

3. Nishi M. Gastric cancer surgery based on clinico-pathological features. Tokyo: Nakayama Institute of Cancer Research; 1994. p. $72-6$. 
4. Kodama M, Koyama K. Indications for pylorus preserving gastrectomy for early gastric cancer located in the middle third of the stomach. World J Surg 1991;15:628-34.

5. Maki T, Shiratori T, Hatafuku T, Sugawara K. Pylorus-preserving gastrectomy as an improved operation for gastric ulcer. Surgery 1967;61:838-45.

6. Isozaki H, Okajima K, Momura E, Ichinona T, Fujii K, Izumi N, et al. Postoperative evaluation of pylorus-preserving gastrectomy for early gastric cancer. Br J Surg 1996;83:266-9.

7. Hotta T, Taniguchi K, Kobayashi Y, Johata K, Sahara M, Naka T, et al. Postoperative evaluation of pylorus-preserving procedures compared with conventional distal gastrectomy for early gastric cancer. Surg Today 2001;31:774-9.

8. Imada T, Rino Y, Takahashi M, Hatori S, Tanaka J, Shiozawa M, et al. Gastric emptying after pylorus-preserving gastrectomy in comparison with conventional subtotal gastrectomy for early gastric carcinoma. Surg Today 1998;28:135-8.

9. Nishikawa K, Kawahara H, Yumiba T, Nishida T, Inoue Y, Ito T, et al. Functional characteristics of the pylorus in patients undergoing pylorus-preserving gastrectomy for early gastric cancer. Surgery 2002;131:613-24.

10. Zhang D, Shimoyama S, Kaminishi M. Feasibility of pyloruspreserving gastrectomy with a wider scope of lymphadenctomy. Arch Surg 1998;133:993-7.

11. Imada T, Rino Y, Takahashi M, Suzuki M, Tanaka J, Shinozawa $M$, et al. Postoperative functional evaluation of pyloruspreserving gastrectomy for early gastric cancer compared with conventional distal gastrectomy. Surgery 1998;123:165-70.

12. Tomita R, Takizawa H, Tanjoh K. Physiologic effects of cisapride on gastric emptying after pylorus-preserving gastrectomy for early gastric cancer. World J Surg 1998;22:35-40.

13. Nakane $Y$, Akehira K, Inoue K, Iiyama H, Sato M, Masuya $Y$, et al. Postoperative evaluation of pylorus-preserving gastrectomy for early gastric cancer. Hepatogastroenterology 2000;47:590-5.

14. Kubo M, Sasako M, Gotoda T, Ono H, Fujishiro M, Saito D, et al. Endoscopic evaluation of the remnant stomach after gastrectomy: proposal for a new classification. Gastric Cancer 2002;5:83-9.

15. Folli S, Dente M, Dell'Amore D, Gaudio M, Nanni O, Saragoni L, et al. Early gastric cancer: prognostic in 223 patients. Br J Surg 1995;82:952-6.

16. Seto Y, Yamaguchi H, Shimoyama S, Shimizu N, Aoki F, Kaminishi M. Results of local resection with regional lymphadenectomy for early gastric cancer. Am J Surg 2001;182:498501.
17. Nakane Y, Michiura T, Inoue K, Sato M, Nakai K, Yamamichi K. Length of the antral segment in pylorus-preserving gastrectomy. Br J Surg 2002;89:220-4.

18. Hobsley M. Dumping and diarrhea. Br J Surg 1981;68:681-4.

19. van der Kleij FGH, Vecht J, Lamers CBHW, Masclee AAM. Diagnostic value of dumping provocation in patients after gastric surgery. Scand J Gastroenterol 1996;31:1162-6.

20. Eagon JC, Miedema BW, Kelly KA. Postgastrectomy syndromes. Surg Clin North Am 1992;72:445-65.

21. Miwa K, Hattori T, Miyazaki I. Duodenogastric reflux and foregut carcinogenesis. Cancer 1995;75:1426-32.

22. Byrne JP, Attwood SE. Duodenogastric reflux and cancer. Hepatogastroenterology 1999;46:74-85.

23. Sugiyama Y, Sohma H, Ozawa M, Hada R, Mikami Y, Konn M, et al. Regurgitant bile acids and mucosal injury of the gastric remnant after partial gastrectomy. Am J Surg 1987;153:399403.

24. Borie F, Plaisant N, Millat B, Hay JM, Fagniez PL, De Saxce B, et al. Treatment and prognosis of early multiple gastric cancer. Eur J Surg Oncol 2003;29:511-4.

25. Takeshita K, Tani M, Honda T, Saeki I, Kando F, Saito N, et al. Treatment of primary multiple early gastric cancer: from the viewpoint of clinicopathologic features. World J Surg 1997;21: 832-6.

26. Seto Y, Nagawa H, Muto T. Treatment of multiple early gastric cancer. Jpn J Clin Oncol 1996;26:134-8.

27. Yamagiwa H, Yoshimura H, Matsuzaki O, Ishihara A. Pathological study of multiple gastric carcinoma. Acta Pathol Jpn 1980;30: 421-6.

28. Bearzi I, Ranaldi R. Multifocal early gastric cancer: morphology and histogenesis. Pathol Res Pract 1986;181:144-7.

29. Honmyo U, Misumi A, Murakami A, Haga Y, Akagi M. Clinicopathological analysis of synchronous multiple gastric carcinoma. Eur J Surg Oncol 1989;15:316-21.

30. Kosaka T, Miwa K, Yonemura Y, Urade M, Ishida T, Takegawa $\mathrm{S}$, et al. A clinicopathological study on multiple gastric cancers with special reference to distal gastrectomy. Cancer 1990;65:26025.

31. Karpas CM, Payson BA, Rechtschaffen J. Intestinal metaplasia and multicentric carcinoma of stomach. NY State J Med 1971;71: $1190-5$.

32. Takeda J, Toyonaga A, Koufuji K, Kodama I, Aoyagi K, Yano S, et al. Early gastric cancer in the remnant stomach. Hepatogastroenterology 1998;45:1907-11. 